\title{
The role of diffuse optical spectroscopy in the clinical management of breast cancer
}

\author{
Natasha Shah ${ }^{\mathrm{a}}$, Albert E. Cerussi ${ }^{\mathrm{a}}$, Dorota Jakubowski ${ }^{\mathrm{a}}$, David Hsiang ${ }^{\mathrm{b}}$, John Butler ${ }^{\mathrm{b}}$ and \\ Bruce J. Tromberg ${ }^{\mathrm{a}, \mathrm{c}, *}$ \\ ${ }^{a}$ Laser Microbeam and Medical Program (LAMMP), Beckman Laser Institute, University of California, Irvine, USA \\ ${ }^{\mathrm{b}}$ Chao Family Comprehensive Cancer Center and Department of Surgical Oncology, University of California, \\ Irvine, Medical Center, USA \\ ${ }^{\mathrm{c}}$ Department of Biomedical Engineering, University of California, Irvine, USA
}

\begin{abstract}
Diffuse optical spectroscopy (DOS) of breast tissue provides quantitative, functional information based on optical absorption and scattering properties that cannot be obtained with other radiographic methods. DOS-measured absorption spectra are used to determine the tissue concentrations of deoxyhemoglobin $(\mathrm{Hb}-\mathrm{R})$, oxyhemoglobin $\left(\mathrm{Hb}-\mathrm{O}_{2}\right)$, lipid, and water $\left(\mathrm{H}_{2} \mathrm{O}\right)$, as well as to provide an index of tissue hemoglobin oxygen saturation $\left(\mathrm{S}_{\mathrm{t}} \mathrm{O}_{2}\right)$. Tissue-scattering spectra provide insight into epithelial, collagen, and lipid contributions to breast density. Clinical studies of women with malignant tumors show that DOS is sensitive to processes such as increased tissue vascularization, hypoxia, and edema. In studies of healthy women, DOS detects variations in breast physiology associated with menopausal status, menstrual cycle changes, and hormone replacement. Current research involves using DOS to monitor tumor response to therapy and the co-registration of DOS with magnetic resonance imaging. By correlating DOS-derived parameters with lesion pathology and specific molecular markers, we anticipate that composite "tissue optical indices" can be developed that non-invasively characterize both tumor and normal breast-tissue function.
\end{abstract}

\section{Basic principles of near-infrared diffuse optical spectroscopy}

Diffuse optical spectroscopy (DOS) utilizes nearinfrared (NIR) light to non-invasively quantify the biochemical composition of breast tissue. The unique information obtained by DOS makes it complementary to conventional radiological techniques and suitable for a variety of clinical applications, such as therapeutic monitoring, lesion characterization, and risk assessment.

NIR propagation in tissue is governed by light absorption, and NIR scattering is governed by endogenous molecules and structures. Light is absorbed by several chromophores of biochemical significance, in-

* Corresponding author: Bruce J. Tromberg, Laser Microbeam and Medical Program (LAMMP), Beckman Laser Institute, University of California, Irvine, CA 92612, USA. Tel.: +1 949824 8367; Fax: +1949824 8413; E-mail: tromberg@bli.uci.edu. cluding hemoglobin, water, and lipid. The spectral characteristics of heme allow for resolution of deoxyhemoglobin (Hb-R) from oxyhemoglobin $\left(\mathrm{Hb}-\mathrm{O}_{2}\right)$. Complete spectra of these principal NIR tissue absorbers are shown in Fig. 1. Shorter wavelengths (ultraviolet and visible) are strongly absorbed by melanin, proteins, and hemoglobin; wavelengths longer than $\sim 1000 \mathrm{~nm}$ are strongly absorbed by water [1]. Thus, weakly absorbed light in the $\sim 650$ - to $\sim 1000$-nm spectral region that can penetrate several centimeters is used for DOS of thick tissues such as breast, brain, and muscle [2].

In addition to absorption, NIR light propagation in tissue is attenuated due to scattering. Photons are scattered strongly as they encounter inhomogeneities in tissue structure and composition caused by changes in refractive index within and between cells. Consequently, NIR light propagation in tissue approximates a diffusive process dominated by multiple scattering events that effectively increase the optical pathlength by as much 10-20 times the linear distance between light 


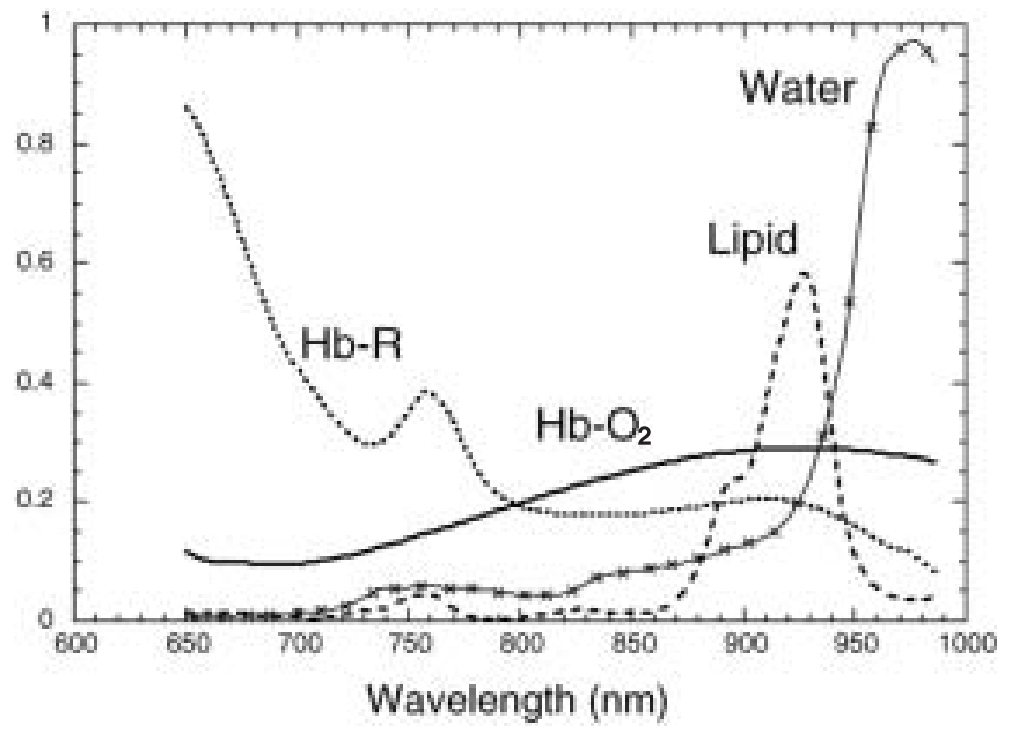

Fig. 1. Absorption spectra of the dominant near-infrared chromophores found in breast tissue: oxyhemoglobin ( $\mathrm{mm}^{-1}, \mathrm{mM}^{-1}$; thick line), deoxyhemoglobin $\left(\mathrm{mm}^{-1}, \mathrm{mM}^{-1}\right.$; dotted line $)$, lipid $\left(\mathrm{mm}^{-1}, \mathrm{~kg}^{-1}\right.$; dashed line) and water $\left(\mathrm{mm}^{-1}\right.$; thin line).

source and detector. Because of multiple light scattering, quantitative, independent measurements of tissue absorption $\left(\mu_{a}\right)$ and reduced scattering $\left(\mu_{s}^{\prime}\right)$ parameters, typically at multiple wavelengths, are necessary for determining the absolute tissue concentration of the principal biological chromophores (hemoglobin, water, and lipid). This can be accomplished at depth in thick tissues using time- or frequency-domain photon migration techniques.

Optical methods applied to breast imaging in the 1980s consisted of trans-illumination and diaphanography, which used continuous-wave visible and NIR light, respectively, to visualize lesions [3]. These qualitative approaches did not account for the strongly scattering nature of NIR light propagation in tissue that led to high false negative and false positive rates and contradictory findings [4-9]. Advances in the understanding of light transport through turbid media during the 1990s led to the development of technologies based on DOS and diffuse optical imaging (DOI). These quantitative approaches have several advantages over earlier continuous-wave techniques [10,11].

The DOS technical strategy we employ in our studies, frequency-domain photon migration (FDPM), uses intensity-modulated light to quantify $\mu_{a}$ and $\mu_{s}{ }^{\prime}$ parameters at discrete wavelengths. The theory and instrumentation used in FDPM are described in detail elsewhere [12-14]. We use a bedside-capable instrument equipped with 10 laser diodes operating between 650-1000 nm (Fig. 2a). The source-modulation fre- quencies are swept from $50-600 \mathrm{MHz}$ [15]. In addition, we employ a steady-state (SS) spectroscopic system to measure $\mu_{a}$ values where FDPM wavelengths do not exist [16]. Together, these techniques provide complete NIR tissue absorption and scattering spectra. Measurements are made by scanning a hand-held probe equipped with optical fibers and an avalanche photodiode detector across the breast (Fig. 2b). The total measurement time to generate complete NIR absorption and scattering spectra $(650-1000 \mathrm{~nm})$ from a single probe typically position is $30-45$ seconds.

Once the spectral information is generated, the tissue concentrations of $\mathrm{Hb}-\mathrm{R}, \mathrm{Hb}-\mathrm{O}_{2}$, lipid, and water are calculated using their wavelength-dependent molar extinction coefficients. Other NIR tissue absorbers such as myoglobin and cytochrome are assumed to have a negligible contribution to light absorption in breast tissue. Quantification of biochemical markers is important to clinical applications because they reveal critical aspects of tissue structure and function. For example, total hemoglobin concentration $([\mathrm{THC}]=[\mathrm{Hb}-$ $\left.\mathrm{R}]+\left[\mathrm{Hb}-\mathrm{O}_{2}\right]\right)$ and tissue hemoglobin oxygenation saturation $\left(\left(\left[\mathrm{Hb}-\mathrm{O}_{2}\right] /[\mathrm{THC}]\right) \times 100 \%\right)$ are composite indices that report on tissue metabolism, vascularization, and angiogenesis. The relative concentrations of lipid vs. water vary with glandular content, hormonal stimulation, and edema. The wavelength-dependence of scattering can be fit to a mathematical model that provides insight into the size and distribution of biological scatterers in tissue $[17,18]$, factors that vary with 


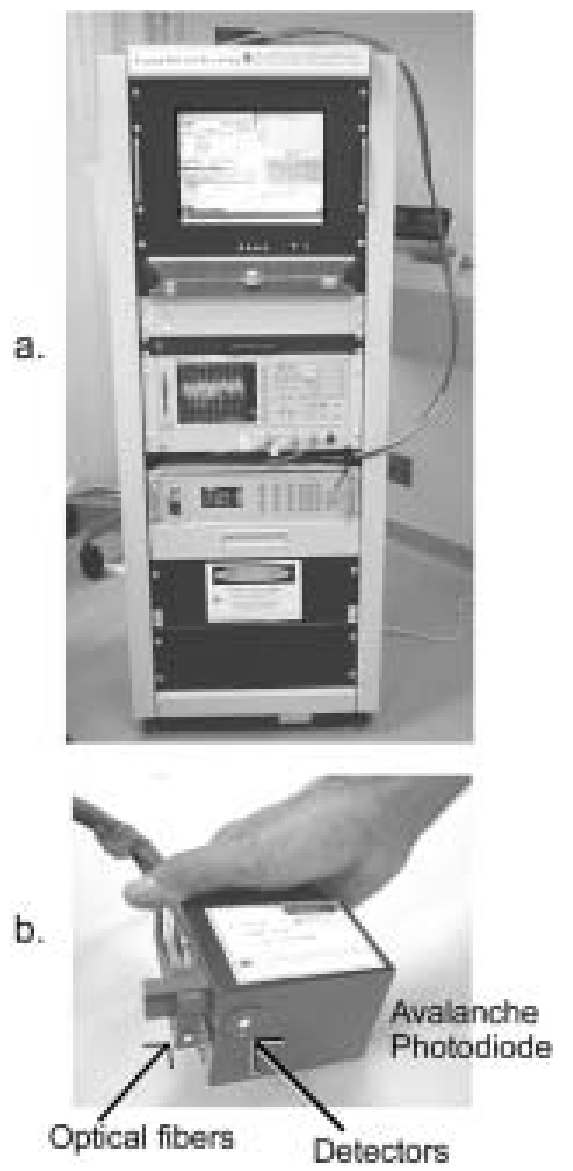

Fig. 2. a. A photograph of the Steady-State Frequency-Domain Photon Migration (SSFDPM) instrument. b. Surface of the hand-held probe that is in contact with the subject and houses the source fibers, a spectrometer fiber, and the avalanche photodiode.

epithelial, lipid, and collagen content $[19,20]$. Thus, the spectral dependence of $\mu_{s}{ }^{\prime}$ and $\mu_{a}$ together noninvasively probe important quantitative functional information that can reveal a wide range of breast-tissue physiological states.

\section{Advantages and limitations of DOS vs. competing technologies}

Diagnostic methods currently in use, such as x-ray mammography, magnetic resonance imaging (MRI), and ultrasound (US), offer excellent anatomic lesiondetection capabilities but generally cannot provide quantitative functional information vital for diagnostic purposes [21]. In addition, MRI and US are used only as secondary procedures to mammography due to factors such as high cost and poor specificity (MRI) or low sensitivity (US). Positron emission tomography (PET) is capable of evaluating tissue metabolic demand but has limited resolution and requires exogenous radionuclides. Consequently, invasive procedures such as fineneedle aspiration or surgical biopsy are implemented to provide a definitive diagnosis.

DOS has potential for clinical impact because it can be a portable, inexpensive, bedside technology that does not require compression and is sensitive intrinsically to the principal components of breast tissue. DOS also is compatible with the use of exogenous probes, such as NIR molecular beacons, and can monitor multiple probes and hemoglobin dynamics simultaneously. Because DOS is responsive to tissue physiology, its performance is not necessarily compromised by structural changes that impact breast density. Methods such as X-ray mammography and MRI have diminished sensitivity and decreased efficacy in women with high breast density [22-24]. As a result, DOS is advantageous for populations with dense breasts, such as younger women and women who receive hormone replacement therapy. Because NIR light is non-ionizing, DOS also is suitable for high-risk populations and can be used to monitor physiological changes on a frequent basis without exposing the tissue to potentially harmful radiation. The primary limitation of DOS is related to the fact that light propagates diffusely in tissue [11]. The volume of tissue probed is dependent upon the optical properties of the tissue, the distance between the light source and detector, and the source modulation frequency. For data reported in this paper, an average volume of approximately $0.5 \mathrm{~cm}^{3}$ is interrogated per measurement. DOS sensitivity is dependent upon the fraction of signal resulting from light probing diseased tissue versus surrounding healthy tissue. Thus, the sensitivity of DOS techniques depends upon the depth and size of the lesion. In addition, DOS methods do not provide the structural resolution obtainable with mammography or MRI. Thus, DOS is not likely to be suitable for screening or detection of structural changes associated with small lesions $(<0.5 \mathrm{~cm})$ or microcalcifications. In some cases, these limitations can be addressed by the use of highly specific exogenous molecular probes.

\section{Sensitivity of DOS to breast tissue physiology and cancer}

Several research groups have demonstrated the sensitivity of DOS to markers of breast disease. For ex- 

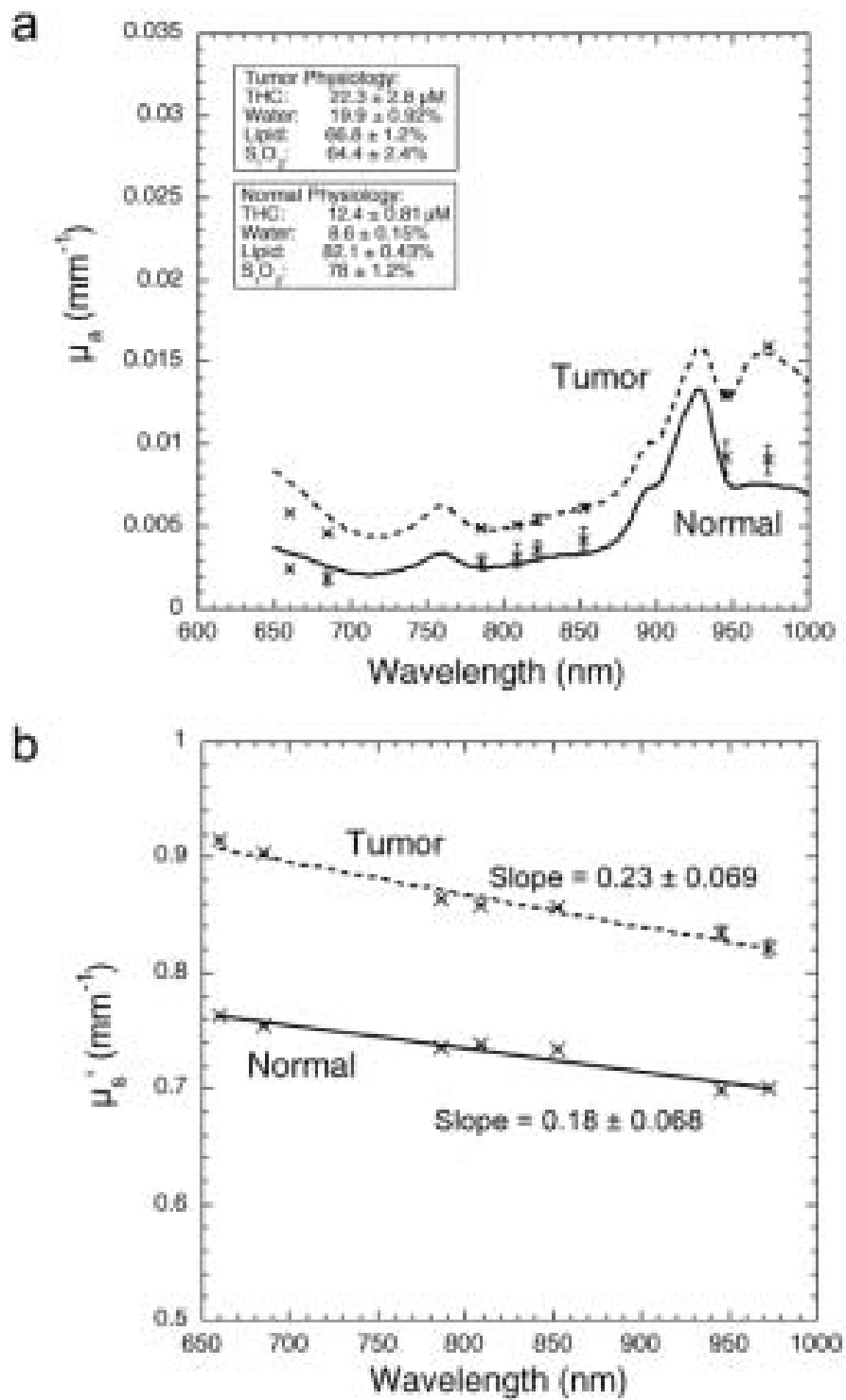

Fig. 3. a. Tissue-absorption coefficient $\left(\mu_{a}\right)$ and b. tissue-scattering coefficient $\left(\mu_{s}{ }^{\prime}\right)$ of cancerous (dashed lines) and normal (solid lines) tissue in a post-menopausal woman. Cancerous tissue displays increased levels of hemoglobin and water as well as a decrease in hemoglobin saturation relative to healthy breast tissue.

ample, in diseased tissue, measurements of tumor total hemoglobin concentration (THC) typically are 2-4 fold greater than in normal tissue, and tumor $\mathrm{S}_{\mathrm{t}} \mathrm{O}_{2}$ values generally are reduced by $5-20 \%[21,25,26]$. Water content can be a reliable indicator of lesions such as fibroadenomas [27]. Because the intensity of light attenuates exponentially with distance, reported tumor spectroscopic values represent a weighted average of lesion and healthy tissue and, thus, tumor/normal contrast depends upon tumor size and depth. Figures $3 \mathrm{a}$ and $3 \mathrm{~b}$ display DOS-measured absorption and scatter- ing spectra of tumor and normal tissue acquired from a 62 -year-old post-menopausal woman with a $\sim 3.0 \mathrm{~cm}$ invasive ductal carcinoma approximately $1 \mathrm{~cm}$ beneath the skin. The symbols indicate $\mu_{a}$ and $\mu_{s}{ }^{\prime}$ values at discrete FDPM wavelengths. The corresponding concentrations of spectroscopically derived parameters are shown in the figure. The tumor tissue displays increased absorption in the $650-850 \mathrm{~nm}$ spectral range, corresponding to higher THC. In addition, the spectral shapes between the two tissue types vary markedly from $900-1000 \mathrm{~nm}$. The lipid peak at $920 \mathrm{~nm}$ is compa- 


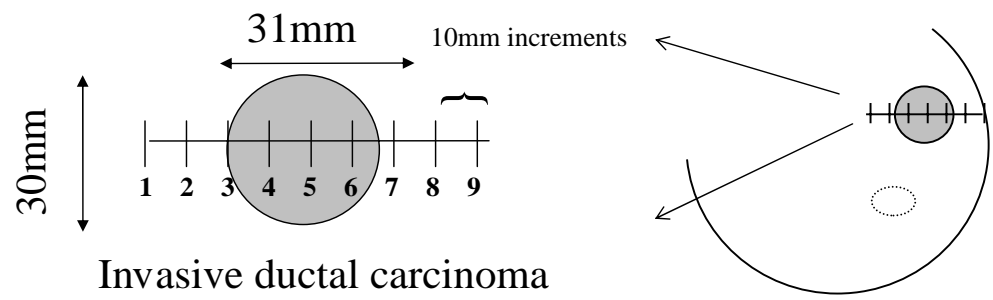

Fig. 4. Diagram of measurement line-scan. The nine measurements were made at $1.0-\mathrm{cm}$ intervals in identical regions on each breast, covering a tissue surface of $8 \mathrm{~cm}$. The center of the line-scan corresponds to the center of the lesion determined by palpation. The lesion was located in the right upper/outer quadrant.

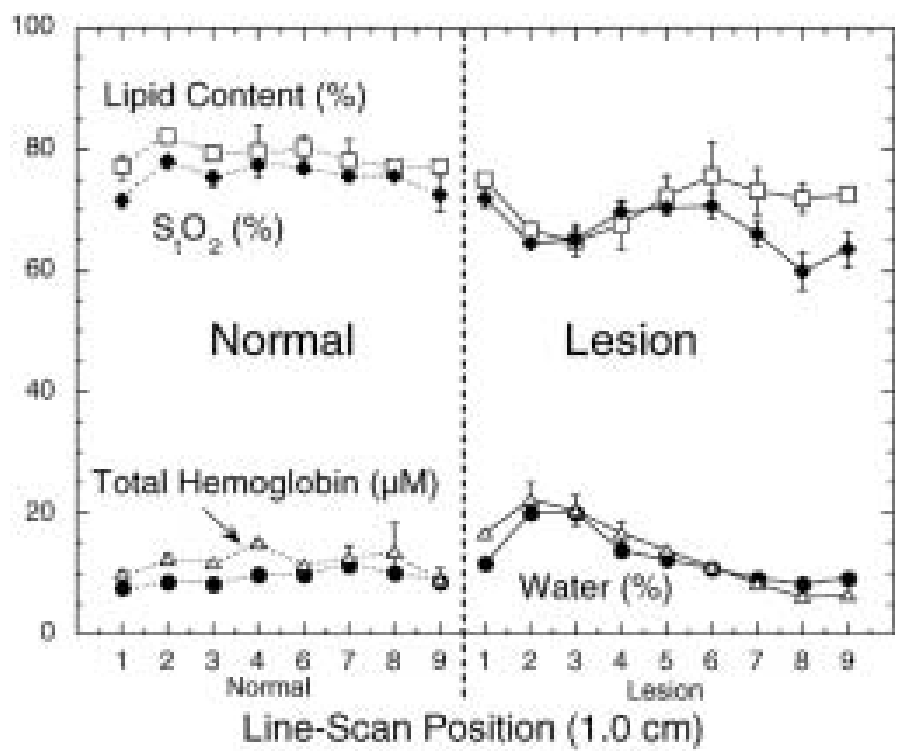

Fig. 5. Total hemoglobin concentration, lipid content, water concentration, and tissue saturation based on position for a 62 -year-old post-menopausal woman with $3.1 \times 3.1 \times 3.0 \mathrm{~cm}$ invasive ductal carcinoma.

rable to the $960 \mathrm{~nm}$ water peak for the tumor. However, the lipid/water ratio is substantially greater for normal tissue, which corresponds to a significant increase in tumor water content. Figure $3 \mathrm{~b}$ shows that this tumor tissue has higher scattering values and a steeper scattering slope than does normal tissue. This suggests that the tumor is composed of smaller scattering particles compared to the surrounding, principally fatty, tissue. The physiological interpretation of this observation is that there is greater epithelial and collagen content in the tumor. Overall, the differences in spectra between the two tissue types are manifestations of multiple physiological changes associated with increased vascularization, cellularity, oxygen consumption, and edema in the tumor.

Clinical measurements are performed by scanning the probe across the lesion and repeating this "linescan" pattern on the contralateral breast (Fig. 4). Re- sults for malignant tumors typically reveal a combination of elevated water and THC and decreased $\mathrm{S}_{\mathrm{t}} \mathrm{O}_{2}$ and lipid content. For illustration, physiological parameters derived from the line-scan across normal and tumor tissue of the patient with an invasive ductal carcinoma are plotted in Fig. 5. These DOS measurements have underlying physiological meaning: high THC corresponds to elevated tissue blood volume fraction, high water content suggests edema and increased cellularity, decreased lipid content reflects displacement of parenchymal adipose, and decreased $\mathrm{S}_{\mathrm{t}} \mathrm{O}_{2}$ indicates increased tissue oxygen metabolism. These functional changes can act in concert to reveal disease. For example, a simple composite clinical index that exploits DOS-derived parameters to describe the tissue metabolic state can be derived. Figure 6 displays one type of "tissue optical index (TOI)," TOI = 


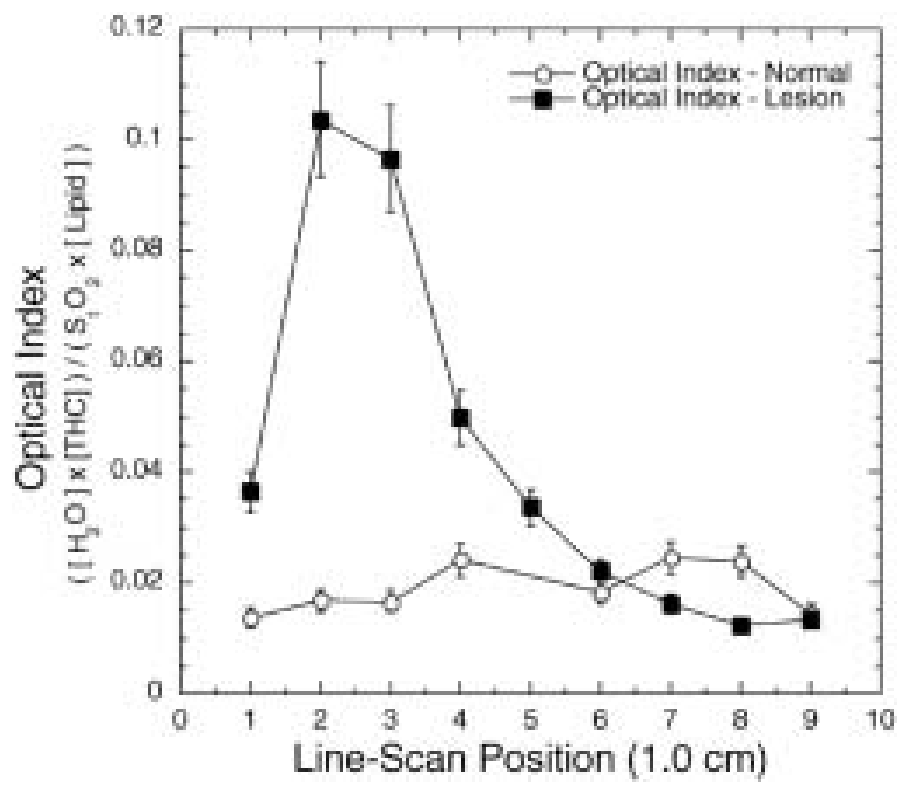

Fig. 6. Tissue optical index for the same subject and measurement pattern shown in Fig. 4. Optical index $=\left([\mathrm{THC}]\left[\mathrm{H}_{0}\right]\right) /\left(\mathrm{S}_{\mathrm{t}} \mathrm{O}_{2}[\mathrm{Lipid}]\right)$ with the units of $\mu \mathrm{M}$.

([water] $[\mathrm{THC}]) /\left(\mathrm{S}_{\mathrm{t}} \mathrm{O}_{2}\right.$ [lipid]), in which elevated TOI values suggest high metabolic activity.

A common procedure for optical methods has been to compare the optical properties of diseased tissue to healthy breast tissue in the same subject. However, the physiology of healthy breast tissue is complex and is influenced by multiple factors such as menstrual cycle, menopause, hormones/drugs, lactation, and pregnancy [20]. DOS-measured parameters are sensitive to the resulting biological changes in the tissue, such as glandular atrophy, glandular metabolism, and changes in collagen-to-fat ratio. Knowledge of the normal values of NIR chromophores will play an important role in evaluating the sensitivity and accuracy of optical methods for detecting and characterizing lesions in the breast.

Figures $7 \mathrm{a}$ and $7 \mathrm{~b}$ display the absorption and scattering spectra of a post- and pre-menopausal woman, respectively [28]. The symbols correspond to $\mu_{a}$ and $\mu_{s}^{\prime}$ values at FDPM wavelengths. The concentrations of spectroscopically derived parameters are shown in the figure. The pre-menopausal woman has increased absorption in the $650-850 \mathrm{~nm}$ spectral region, corresponding to higher THC. In addition, the premenopausal woman has an elevated water absorption peak at $960 \mathrm{~nm}$; whereas the post-menopausal woman displays strong lipid absorption at $930 \mathrm{~nm}$, with little or no features at $960 \mathrm{~nm}$. These spectral differences between the two women reflect physiological changes due to glandular atrophy after menopause. Other studies confirm these trends $[29,30]$. Since glandular tissue is more vascularized than the surrounding parenchyma, a post-menopausal drop in total hemoglobin is expected, without a considerable change in $\mathrm{S}_{\mathrm{t}} \mathrm{O}_{2}$. The slope of the scattering spectrum ("scatter power") has been related to the average size of the scattering particle [17] and can provide insight into the structure and composition of the breast, such as the collagen-to-fat ratio [28]. After menopause, small-particle glandular tissue is replaced by fat (relatively large particles), which can lead to a reduction in scatter power.

These trends persist in a population of women. Figure 8 displays the average values of each parameter based on menopausal status for 21 healthy volunteers (14 pre-menopausal and 7 post-menopausal women). Thus, DOS measurements are sensitive to biological processes in the breast that occur with menopause, such as decreased fibroglandular volume, blood flow, and metabolism [31-33]. Previous studies [34,35] show that DOS also is intrinsically sensitive to breasttissue variations during the menstrual cycle of premenopausal women. Results in a limited number of cases have shown that $\mu_{a}$ is higher before the onset of menses than prior to ovulation, which translates into an increase in tissue hemoglobin and water content. These changes are consistent with the physiological effects due to ovarian hormone fluctuations during the menstrual cycle [36,37]. 

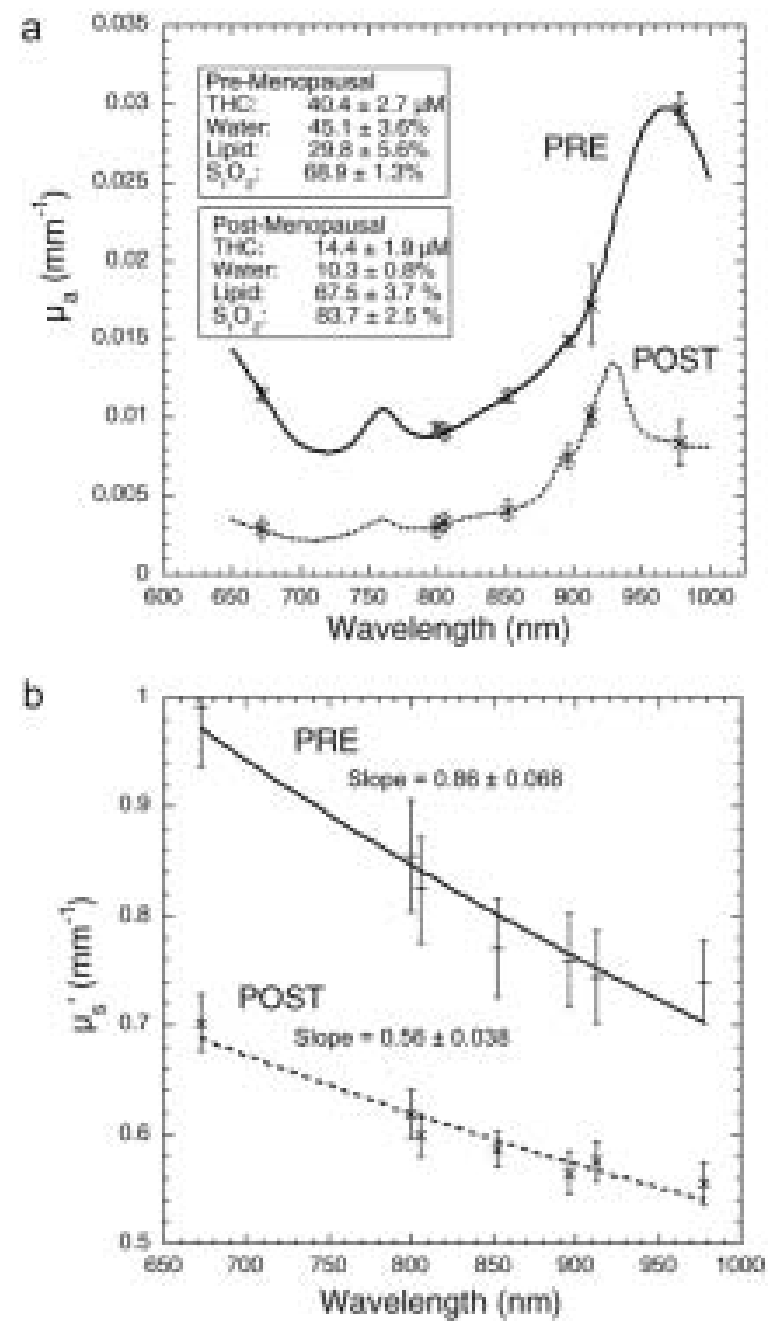

Fig. 7. a. Tissue-absorption coefficient $\left(\mu_{a}\right)$ and b. tissue-scattering coefficient $\left(\mu_{s}^{\prime}\right)$ of normal breast tissue in a 54-year-old postmenopausal woman and a 32-year-old pre-menopausal woman.

\section{Current research}

\subsection{Response to cancer therapies}

Currently, much effort in the radiology community is directed toward imaging angiogenesis, a key enabling event in tumor growth and metastasis [38]. DOS is highly sensitive to changes in hemoglobin content and oxygen utilization at the micro-vessel level, processes that are associated with angiogenesis. Ongoing studies in our laboratory and in other research groups [25,26, 39-41] have shown excellent correlation between several DOS-derived hemoglobin parameters and tumor pathology. Assessment of tumor angiogenesis and ves-

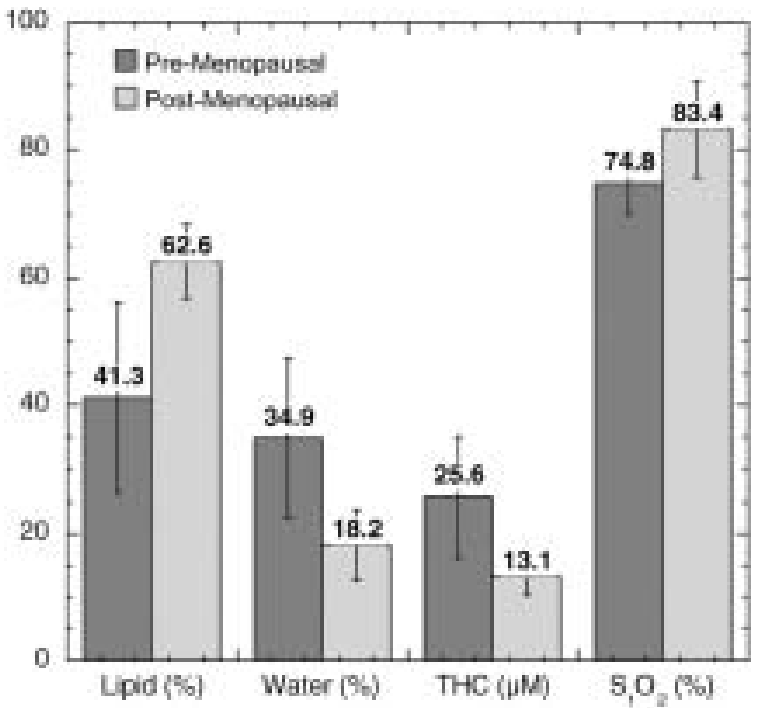

Fig. 8. Average values of total hemoglobin, tissue oxygen saturation, water, and lipid content for pre-menopausal women $(n=14)$ and post-menopausal women $(n=7)$.

sel density can be applied to monitoring tumor response to therapy and predicting therapeutic outcome.

One therapeutic approach for women with locally advanced breast cancer is pre-surgical (neo-adjuvant) chemotherapy. This strategy can shrink tumors and allow for more complete surgical removal; thereby increasing long-term survival [42]. Determining the optimal chemotherapy regimen and duration is challenging because of the difficulty associated with quantitatively evaluating therapeutic response. We are exploring the use of non-invasive optical methods for rapid assessment of tumor physiology in order to maximize therapeutic efficacy. Preliminary results using DOS to monitor patients who receive neo-adjuvant therapy have shown excellent sensitivity to tumor response, functional changes, size, and residual disease via hemoglobin and water signatures [43]. For example, Fig. 9 shows that, during the first week of doxorubicin/cyclophosphamide therapy, peak tumor THC levels dropped by $27 \%$, whereas normal breast tissue and abdomen (control site) changed by $\sim 5 \%$. On day 68, after three cycles of chemotherapy, peak tumor THC was $44 \%$ of pre-chemotherapy levels, and normal breast and abdomen decreased to $\sim 70 \%$ and $\sim 60 \%$, respectively. Evaluating tumor response in terms of tissue optical indices will allow for a more comprehensive assessment of disease status and may provide an early indicator of response to therapy and optimize therapeutic outcome. 


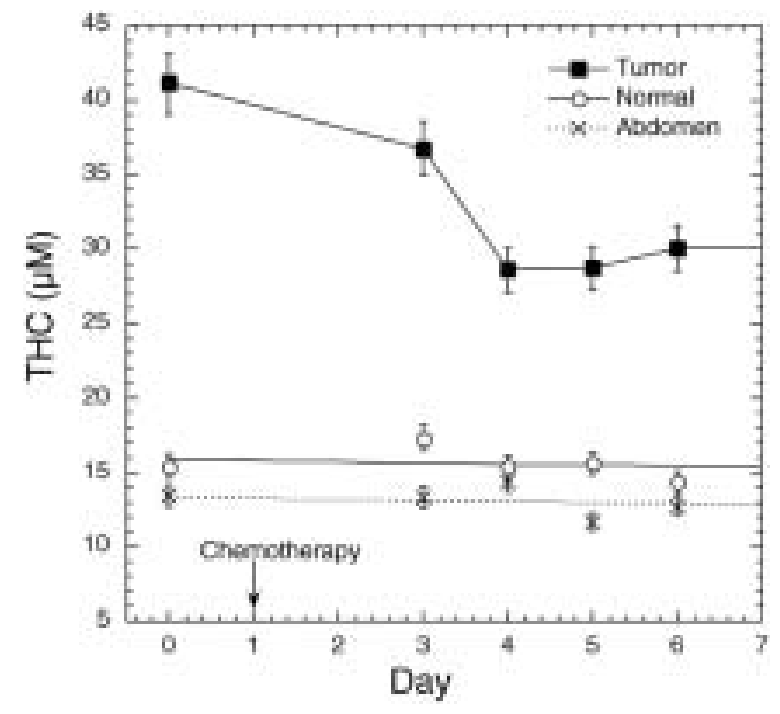

Fig. 9. Tumor response to neo-adjuvant chemotherapy during the first week of treatment. The plot shows total hemoglobin concentration vs. day of treatment, where day 1 indicates the onset of chemotherapy. "Tumor" data points are measurements taken on the center of the lesion. "Normal" indicates measurements taken on the identical position on the contralateral breast, and "abdomen" indicates measurements taken near the navel to represent the systemic response to chemotherapy.

\subsection{Combined DOS and magnetic resonance imaging}

Diffuse optical methods have valuable functional capabilities but are not as effective for disease localization as conventional anatomic imaging techniques. In order to address this limitation, many investigators have developed strategies for combining DOS with MRI [4446] US [41,47], and mammography. Integration of high-resolution structural information from MRI can complement optically derived functional information. A combination of these methods has the potential to enhance our understanding of the complex biological processes that are associated with tumor transformation, growth, and hemodynamics. We have developed a rat tumor model to investigate DOS co-registration with MRI [48,49]. Simultaneous T2-weighted, gadoliniumdiethylenetriaminepentaacetic (Gd-DTPA) contrast-enhanced MRI/DOS measurements of a rat abdomen in cross-section (Figures 10a and 10b) show that contrastenhanced images can separate viable, edematous, and necrotic tumor tissues for correlation with contrastagent information and NIR-DOS measurements. DOS results from 10 rats reveal increasing water content in edematous tissues and decreasing THC and $\mathrm{S}_{\mathrm{t}} \mathrm{O}_{2}$ values in necrotic tissues over a 20-day period [49].
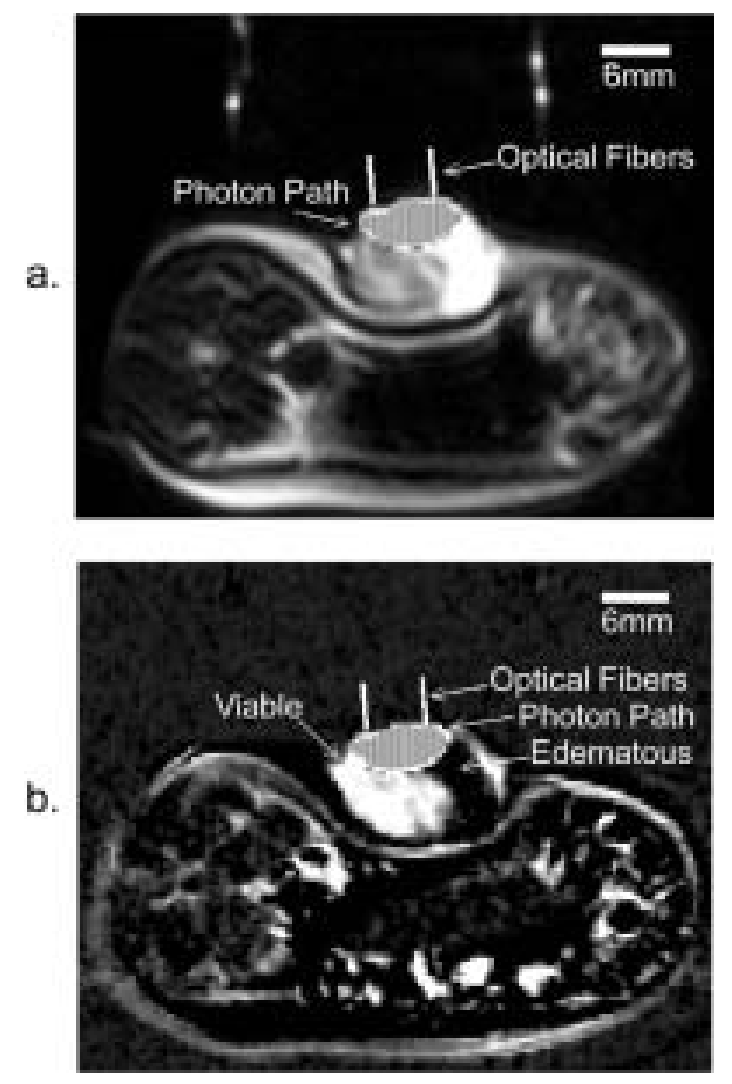

Fig. 10. a. T2-weighted magnetic resonance image of a rat cross-section with human adenocarcinoma. The photon path indicating the tissue sampled by the DOS measurement is superimposed on the image (gray ellipse). Light areas represent tissues with high water content. b. Magnetic resonance image of gadolinium-diethylenetriaminepentaacetic (Gd-DTPA) contrast-enhanced image with superimposed photon path. Light areas represent region of blood flow (viable tissues). Hence, image b shows that the left half of the tumor is viable and the right half is edematous.

\section{Future improvements}

Combining DOS-measured parameters with conventional radiological imaging and advanced immunohistochemical methods will enhance our ability to detect and characterize breast lesions. Future study improvements include correlating optical data with radiographic density, a risk factor for breast disease [50-54]. Radiographic density can be altered by diet, hormone use, and other factors [55-57]; however, mammography is unsuitable for monitoring density changes regularly. DOS quantitatively measures changes in tissue structure via optically derived functional parameters such as tissue "scatter power," water concentration, and lipid content. Consequently, DOS may be appropriate for frequent evaluation of breast density varia- 
tions because it does not utilize ionizing radiation and has a high degree of patient acceptance. (DOS does not require compression). Furthermore, DOS is a relatively inexpensive and simple addition to mammography, US, and MRI. Incorporation of DOS into these modalities will provide unique functional information from both endogenous targets and exogenous probes, although precise co-registration with anatomic images remains an important challenge.

\section{Conclusions}

We have demonstrated that DOS measurements of biochemical composition correlate well with known breast-tissue physiology and tumor pathology. Combining DOS methods with immunohistochemical and radiological information can lead to the development of simple predictive optical indices that can provide specific assessment of tissue blood volume, metabolism, and cellular and matrix density. Ultimately, this approach is expected to advance our understanding of the origins of breast disease, improve tumor characterization, and have a practical impact on optimizing the efficacy of neo-adjuvant chemotherapy.

\section{Acknowledgements}

This work was supported by the National Institutes of Health (NIH) under grants RR01192 (Laser Microbeam and Medical Program: LAMMP) and NIH P20-CA86182; the California Breast Cancer Research Program, and the Avon Foundation-Chao Family Comprehensive Cancer Center. The authors thank the MRI/DOS research group at the University of California at Irvine: (Orhan Nalcioglu, Gultekin Gulsen, Hon Yu, Sean Merritt, David Cuccia, Frederic Bevilacqua, and Anthony J. Durkin) for their contribution to this work. The authors also thank Anirban Mazumdar for his assistance in processing tissue optical measurement data.

\section{References}

[1] B.C. Wilson, W.P. Jeeves and D.M. Lowe, In vivo and postmortem measurements of the attenuation spectra of light in mammalian tissues, Photochem Photobiol 42 (1985), 153162.

[2] B.J. Tromberg, N. Shah, R. Lanning, A. Cerussi, J. Espinoza, T. Pham, L. Svaasand and J. Butler, Non-invasive in vivo characterization of breast tumors using photon migration spectroscopy, Neoplasia 2 (2000), 26-40.
[3] E. Carlson, in: Diagnostic Imaging, S. Spectrascan, C.T. Windsor, eds, 1982, pp. 28.

[4] R.J. Bartrum, Jr. and H.C. Crow, Transillumination lightscanning to diagnose breast cancer: a feasibility study, AJR Am J Roentgenol 142 (1984), 409-414.

[5] H. Wallberg, A. Alveryd, K. Nasiell, P. Sundelin, U. Bergvall and S. Troell, Diaphanography in benign breast disorders. Correlation with clinical examination, mammography, cytology and histology, Acta Radiol Diagn Stockh 26 (1985), 129-136.

[6] N. Bundred, P. Levack, D.J. Watmough and J.A. Watmough, Preliminary results using computerized telediaphanography for investigating breast disease, Br J Hosp Med 37 (1987), 70-71.

[7] B. Drexler, J.L. Davis and G. Schofield, Diaphanography in the diagnosis of breast cancer, Radiology 157 (1985), 41-44.

[8] B. Monsees, J.M. Destouet and D. Gersell, Light scanning of nonpalpable breast lesions: reevaluation, Radiology 167 (1988), 352.

[9] A. Alveryd, I. Andersson, K. Aspegren, G. Balldin, N. Bjurstam, G. Edström, G. Fagerberg, U. Glas, O. Jarlman, S.A. Larsson et al., Lightscanning versus mammography for the detection of breast cancer in screening and clinical practice, A Swedish multicenter study, Cancer 65 (1990), 1671-1677.

[10] M.S. Patterson, B. Chance and B.C. Wilson, Time resolved reflectance and transmittance for the non-invasive measurement of tissue optical properties, Appl Opt 28 (1989), 2331-2336.

[11] J.B. Fishkin and E. Gratton, Propagation of photon-density waves in strongly scattering media containing an absorbing semi-infinite plane bounded by a straight edge, J Opt Soc Am A 10 (1993), 127-140.

[12] A. Yodh and B. Chance, Spectroscopy and imaging with diffusing light, Phys Today 48 (1996), 34-40.

[13] J.B. Fishkin, S. Fantini, M.J. vande Ven and E. Gratton, Gigahertz photon density waves in a turbid medium: theory and experiments, Phys Rev E 53 (1996), 2307-2319.

[14] T.H. Pham, O. Coquoz, J.B. Fishkin, E. Anderson and B.J. Tromberg, Broad bandwidth frequency domain instrument for quantitative tissue optical spectroscopy, Rev Sci Instrum 71 (2000), 2500-2513.

[15] E.M. Sevick, B. Change, J. Leigh, S. Nioka and M. Maris, Quantitation of time-resolved and frequency-resolved optical spectra for the determination of tissue oxygenation, Anal Biochem 195 (1991), 330-351.

[16] F. Bevilacqua, A.J. Berger, A.E. Cerussi, D. Jakubowski and B.J. Tromberg, Broadband absorption spectroscopy in turbid media by combined frequency-domain and steady-state methods, Appl Opt 39 (2000), 6498-6507.

[17] A.M.K. Nilsson, C. Sturesson, D.L. Liu and S. AnderssonEngels, Changes in spectral shape of tissue optical properties in conjunction with laser-induced thermotherapy, Appl Opt 37 (1998), 1256-1267.

[18] J.R. Mourant, T. Fuselier, J. Boyer, T.M. Johnson and I.J. Bigio, Predictions and measurements of scattering and absorption over broad wavelength ranges in tissue phantoms, Appl Opt 36 (1997), 949-957.

[19] B. Beauvoit, T. Kitai and B. Chance, Contribution of the mitochondrial compartment to the optical properties of the rat liver: a theoretical and practical approach, Biophys J 67 (1994), 2501-2510.

[20] S. Thomsen and D. Tatman, Physiological and pathological factors of human breast disease that can influence optical diagnosis, Ann NY Acad Sci 838 (1998), 171-193.

[21] B.J. Tromberg, O. Coquoz, J.B. Fishkin, T. Phan, E.R. Anderson, J. Butler, M. Cahn, J.D. Gross, V. Venugopalan and 
D. Pham, Non-invasive measurements of breast tissue optical properties using frequency-domain photon migration, Philos Trans R Soc Lond B Biol Sci 352 (1997), 661-668.

[22] C.J. Baines and R. Dayan, A tangled web: factors likely to affect the efficacy of screening mammography, J Natl Cancer Inst 91 (1999), 833-838.

[23] K. Kerlikowske and J. Barclay, Outcomes of modern screening mammography, Journal of the National Cancer Institute, Monographs 169 (1997), 105-111.

[24] W.H. Hindle, L. Davis and D. Wright, Clinical value of mammography for symptomatic women 35 years of age and younger, Am J Obstet Gynecol 180 (1999), 1484-1490.

[25] S. Fantini, S.A. Walker, M.A. Franceschini, M. Kaschke, P.M. Schlag and K.T. Moesta, Assessment of the size, position, and optical properties of breast tumors in vivo by noninvasive optical methods, Appl Opt 37 (1998), 1982-1989.

[26] B.W. Pogue, S.P. Poplack, T.O. McBride, W.A. Wells, K.S Osterman, U.L. Osterberg and K.D. Paulsen, Quantitative hemoglobin tomography with diffuse near-infrared spectroscopy: pilot results in the breast, Radiology 218 (2001), 261-266.

[27] A.E. Cerussi, D. Jakubowski, N. Shah, F. Bevilacqua, R. Lanning, A.J. Berger, D. Hsiang, J. Butler, R.F. Holcombe and B.J. Tromberg, Spectroscopy enhances the information content of optical mammography, J Biomed. Opt 7 (2002), 60-71.

[28] A.E. Cerussi, A.J. Berger, F. Bevilacqua, N. Shah, D. Jakubowski, J. Butler, R.F. Holcombe and B.J. Tromberg, Sources of absorption and scattering contrast for near-infrared optical mammography, Acad Radiol 8 (2001), 211-218.

[29] R. Cubeddu, A. Pifferi, P. Taroni, A. Torricelli and G. Valentini, Noninvasive absorption and scattering spectroscopy of bulk diffusive media: an application to the optical characterization of human breast, Appl Phys Lett 74 (1999), 874-876.

[30] K. Suzuki, Y. Yamashita, K. Ohta, M. Kaneko, M. Yoshida and B. Chance, Quantitative measurement of optical parameters in normal breasts using time-resolved spectroscopy: in vivo results of 30 Japanese women, J Biomed Opt 1 (1996), 330 334.

[31] J. Brisson, A.S. Morrison and N. Khalid, Mammographic parenchymal features and breast cancer in the Breast Cancer Detection Demonstration Project, J Nat Cancer Inst (Bethesda) 80 (1988), 1534-1540.

[32] J.O. Drife, Breast modifications during the menstrual cycle, Suppl Int J Gynecol Obstet 1 (1989), 19-24.

[33] D.L. Page and W.D. Dupont, Histopathologic risk factors for breast cancer in women with benign breast disease, Semin Surg Oncol 4 (1988), 213-217.

[34] N. Shah, A. Cerussi, C. Eker, J. Espinoza, J. Butler, J. Fishkin, R. Hornung and B. Tromberg, Noninvasive functional optical spectroscopy of human breast tissue, Proc Natl Acad Sci USA 98 (2001), 4420-4425.

[35] R. Cubeddu, C. D’Andrea, A. Pifferi, P. Taroni, A. Torricelli and G. Valentini, Effects of the menstrual cycle on the red and near-infrared optical properties of the human breast, Photochem Photobiol 72 (2000), 383-391.

[36] P.A. Fowler, C.E. Casey, G.G. Cameron, M.A. Foster and C.H. Knight, Cyclic changes in composition and volume of the breast during the menstrual cycle, measured by magnetic resonance imaging, Br J Obstet Gynaecol 97 (1990), 595-602.

[37] S.J. Graham, P.L. Stanchev, J.O. Lloyd-Smith, M.J. Bronskill and D.B. Plewes, Changes in fibroglandular volume and water content of breast tissue during the menstrual cycle observed by MR imaging at 1.5 T, J Mag Reson Imaging 5 (1995), 695-701.
[38] J. Folkman and K. Beckner, Angiogenesis imaging, Acad Radiol 7 (2000), 783-785.

[39] M.A. Franceschini, K.T. Moesta, S. Fantini, G. Gaida, E. Gratton, H. Jess, W.W. Mantulin, M. Seeber, P.M. Schlag and M. Kaschke, Frequency-domain techniques enhance optical mammography: initial clinical results, Proc Natl Acad Sci USA 94 (1997), 6468-6473.

[40] D. Grosenick, H. Wabnitz, H.H. Rinneberg, K.T. Moesta and P.M. Schlag, Development of a time-domain optical mammograph and first in vivo applications, Appl Opt 38 (1999), 2927-2943.

41] M.J. Holboke, B.J. Tromberg, N. Shah, J. Fishkin, D. Kidney, J. Butler, B. Chance and A.G. Yodh, Three-dimensional diffuse optical mammography with ultrasound localization in a human subject, J Biomed Opt 5 (2000), 237-247.

[42] W. Cance, L. Carey and B. Calvo, Long-term outcome of neoadjuvant therapy for locally advanced breast carcinoma: effective clinical downstaging allows breast preservation and predicts outstanding local control and survival, Ann Surg 236 (2002), 295-303

[43] D. Jakubowski, A.E. Cerussi, F. Bevilacqua, N. Shah, D. Hsiang, J. Butler, R.F. Holcombe and B. Tromberg, Monitoring breast tumor response to chemotherapy with broadband near-infrared tissue spectroscopy, OSA Biomedical Topical Meetings, Advances in Optical Imaging and Photon Migration, Miami, FL, April 7-10, 2002.

[44] J. Chang, H.L. Graber, P.C. Koo, R. Aronson, S.L. Barbour and R.L. Barbour, Optical imaging of anatomical maps derived from magnetic resonance images using time-independent optical sources, IEEE Trans Med Imaging 16 (1997), 68-77.

[45] V. Ntziachristos, A.G. Yodh, M. Schnall and B. Chance, Concurrent MRI and diffuse optical tomography of breast after indocyanine green enhancement, Proc Natl Acad Sci USA 97 (2000), 2767-2772.

[46] V. Ntziachristos, A.G. Yodh, M.D. Schnall and B. Chance, MRI-guided diffuse optical spectroscopy of malignant and benign breast lesions, Neoplasia 4 (2002), 347-354.

[47] Q. Zhu, E. Conant and B. Chance, Optical imaging as an adjunct to sonograph in differentiating benign from malignant breast lesions, J Biomed Opt 5 (2000), 229-236.

[48] D. Cuccia, F. Bevilacqua, A.J. Durkin, S.I. Merritt, B. Tromberg, G. Gulsen, H. Yu, J. Wang and O. Nalcioglu, In-vivo quantification of optical contrast agent dynamics in rat tumors using diffuse optical spectroscopy with MRI coresgistration, Appl Opt, Special Issue: Topics in Biomedical Optics (accepted for publication).

[49] S.I. Merritt, F. Bevilacqua, A.J. Durkin, D. Cuccia, R. Lanning, B. Tromberg, G. Gulsen, H. Yu, J. Wang and O. Nalcioglu, Monitoring tumor physiology using near-infrared spectroscopy and MRI co-registration, Appl Opt, Special Issue: Topics in Biomedical Optics (accepted for publication).

[50] J.W. Byng, M.J. Yaffe, R.A. Jong, R.S. Shumak, G.A. Lockwood, D.L. Tritchler and N.F. Boyd, Analysis of mammographic density and breast cancer risk from digitized mammograms, Radiographics 18 (1998), 1587-1598.

[51] E. Sala, R. Warren, S. Duffy, A. Welch, R. Luben and N. Day, High risk mammographic parenchymal patterns and diet: a case-control study, Br J Cancer 83 (2000), 121-126.

[52] N.F. Boyd, G.A. Lockwood, J.W. Byng, D.L. Tritchler and M.J. Yaffe, Mammographic densities and breast cancer risk, Cancer Epidemio Biomarkers Prev 7 (1998), 1133-1144.

[53] A.F. Saftlas, R.N. Hoover, L.A. Brinton, M. Szklo, D.R. Olson, M. Salane and J.N. Wolfe, Mammographic densities and risk of breast cancer, Cancer 67 (1991), 2833-2838. 
[54] C. Byrne, Mammographic density: a breast cancer risk factor or diagnostic indicator? Acad Radiol 9 (2002), 253-255.

[55] E. Lundstrom, B. Wilczek, Z. von Palffy, G. Soderqvist and B. von Schoultz, Mammographic breast density during hormone replacement therapy: effects of continuous combination, unopposed transdermal and low-potency estrogen regimens, $\mathrm{Cli}$ macteric 4 (2001), 42-48.

[56] J. Brisson, B. Brisson, G. Cote, E. Maunsell, S. Berube and J.
Robert, Tamoxifen and mammographic breast densities, Cancer Epidemiol Biomarkers Prev 9 (2000), 911-915.

[57] N.F. Boyd, C. Greenberg, G. Lockwood, L. Little, L. Martin, J. Byng, M. Yaffe and D. Tritchler, Effects at two years of a low-fat, high-carbohydrate diet on radiologic features of the breast: results from a randomized trial, J Natl Cancer Inst (Bethesda) 89 (1997), 488-496. 


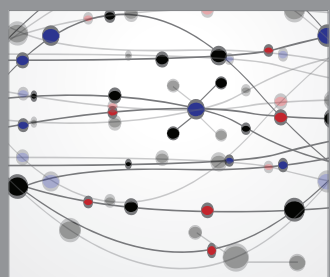

The Scientific World Journal
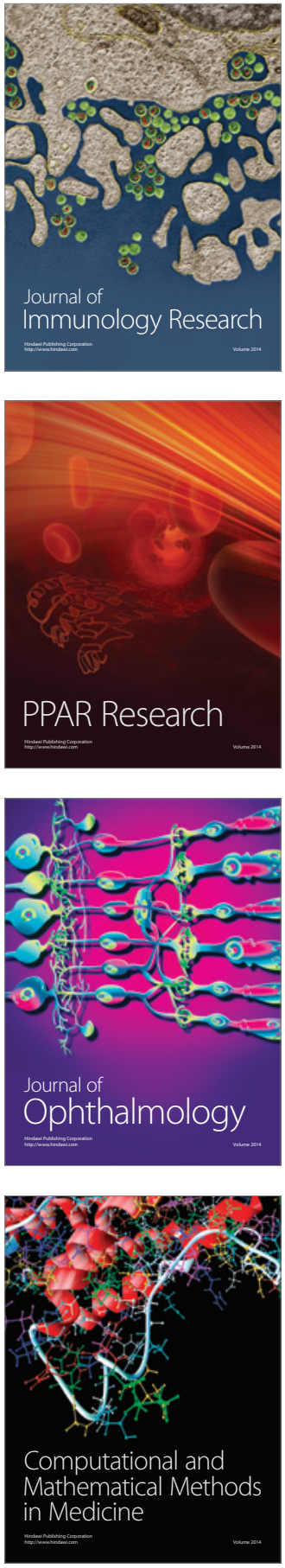

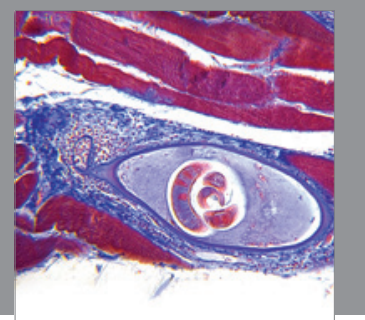

Gastroenterology

Research and Practice
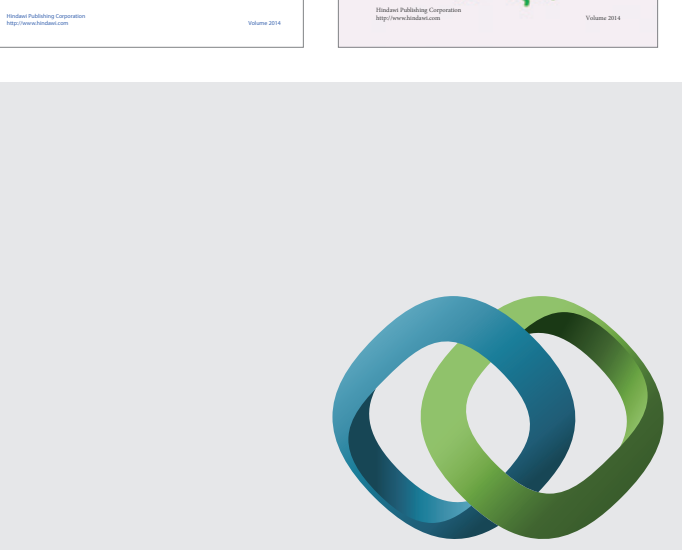

\section{Hindawi}

Submit your manuscripts at

http://www.hindawi.com
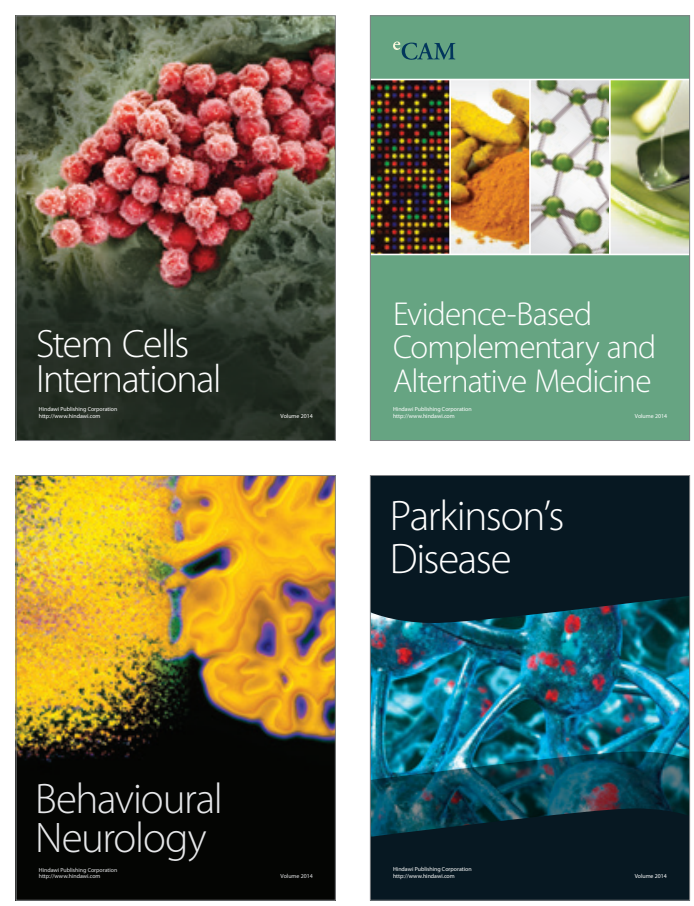

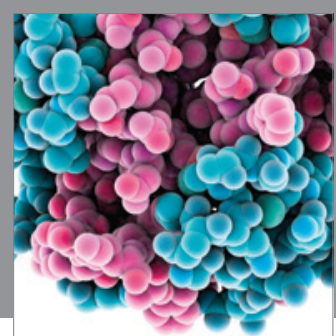

Journal of
Diabetes Research

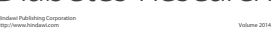

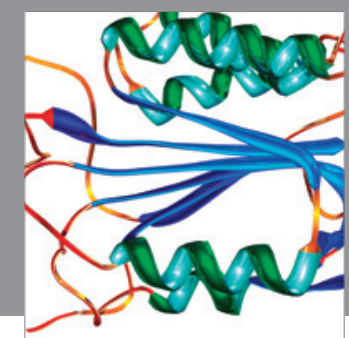

Disease Markers
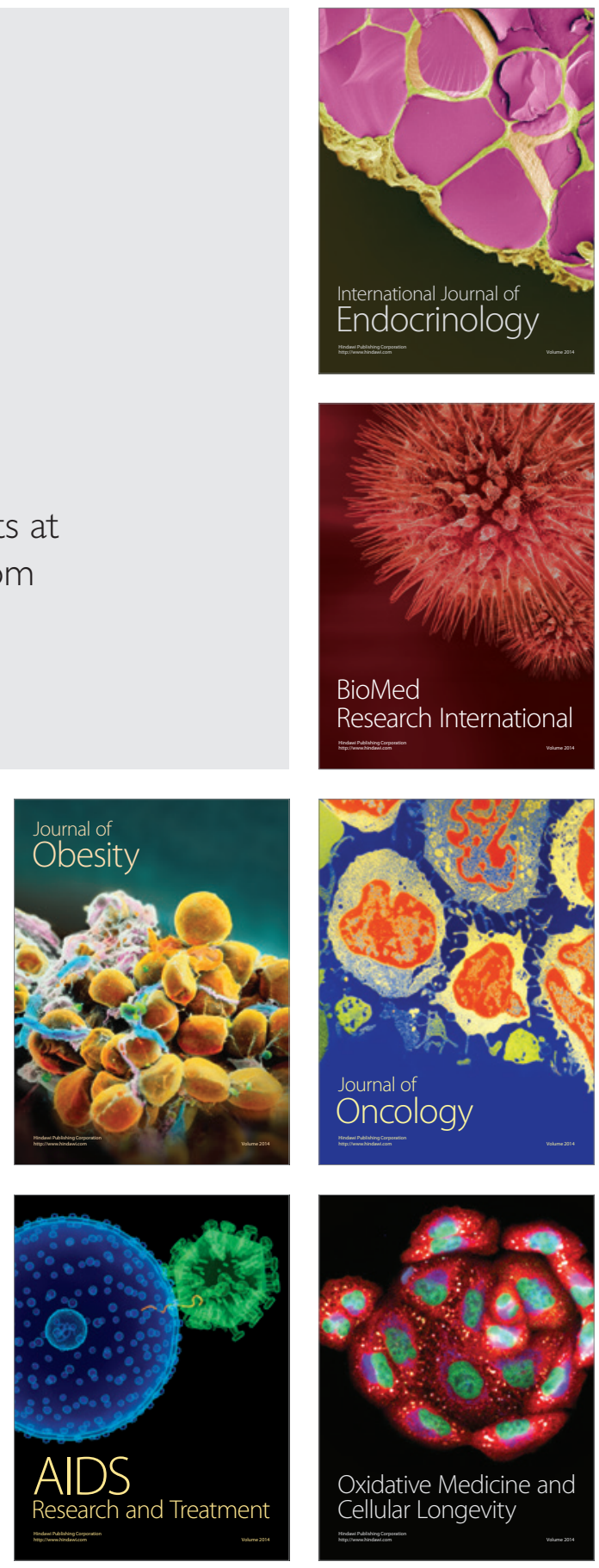